\title{
GMR
}

\section{Variability with altitude of major histocompatibility complex-related microsatellite loci in goats from Southwest China}

\author{
G.X. E, Y.F. Huang, Y.J. Zhao and R.S. Na \\ College of Animal Science and Technology, \\ Chongqing Key Laboratory of Forage \& Herbivores, \\ Chongqing Engineering Research Centre for Herbivores Resource Protection and \\ Utilization, Southwest University, Chongqing, China \\ Corresponding author: Y.F. Huang \\ E-mail: H67738337@swu.edu.cn
}

Genet. Mol. Res. 14 (4): 14629-14636 (2015)

Received June 29, 2015

Accepted September 2, 2015

Published November 18, 2015

DOI http://dx.doi.org/10.4238/2015.November.18.26

ABSTRACT. We aimed to use microsatellite BM1258 loci of the major histocompatibility complex (MHC) as an indicator of the influence of genetic diversity of immunity in goats (Dazu Black, Hechuan White, Meigu, and Tibetan goat). In total, 132 animals comprising 50 Dazu Black goats, 24 Hechuan White goats, 34 Meigu goats, and 24 Tibetan goats were examined. Collectively, 18 different alleles and 42 genotypes were found. The overall observed levels of heterozygosity showed large divergence from the expected levels in the four breeds, and an increase in the mean number of alleles of BM1258 accompanied decreasing altitude of the livestock's habitat. Our results indicate that low-altitude regions or plains were more conducive to genetic material exchange and gene flow between different populations. In addition, it seems that the breeds from low-altitude regions were less susceptible to problems introduced by commercial animals.

Key words: Microsatellite; BM1258; Major histocompatibility complex; Chinese indigenous goats; Altitude 


\section{INTRODUCTION}

The goat (Capra hircus) is a predominant domestic animal; it not only provides meat, but also cashmere, milk, wool, and fur. Currently, a wide variety of goat breeds with abundant phenotypic diversity exists because of domestication and selective breeding. However, commercial lines and industrialized livestock production systems have spread over all continents resulting in a decrease in large indigenous goat breeds in comparison with some commercial breeds. Therefore, it seems particularly important to build a complete system for monitoring genetic resource conservation in goats. In addition, indigenous goat breeds are often well adapted to harsh local environmental conditions, and these adaptations contribute to a growing interest in indigenous species for conservation and immunogenic diversity.

Major histocompatibility complex (MHC) genes play important roles in the immune system, autoimmunity, reproduction, economic traits, and life history strategies. Candidate genes for the study of disease ecology and evolution have also been found within the MHC genomic region (Edidin, 1988). In mammals, MHC genes are located in a gene-dense genomic region that includes similar members of several gene families and contains the most polymorphic functional genes in vertebrate genomes (Satta et al., 1998; The MHC Sequencing Consortium et al., 1999; Gaudieri et al., 2000). Their products recognize exogenous peptides and present them to specialist immune cells to initiate an immune response (Klein and Figueroa, 1986), In addition, specific MHC haplotypes and MHC heterozygosity are associated with immunity to diseases (de Bakker et al., 2006; Gregersen et al., 2006; Oliver et al., 2009; Kloch et al., 2010; Westerdahl et al., 2012; Grossen et al., 2014). Therefore, the higher allelic diversity at the MHC region is expected to be favored because individuals with a broader range of $\mathrm{MHC}$ sequences (binding and presenting a broader range of pathogenic peptides) should be more successful at fighting diseases (Gregersen et al., 2006; Kloch et al., 2010; Westerdahl et al., 2012).

As DNA-based genetic markers, microsatellites have been widely used as a standard technique for molecular genetic evaluation and mapping of the goat genome (Rout et al., 2008; Rout et al., 2012; Vahidi et al., 2014), particularly in China (Li et al., 2002; Wei et al., 2014). Although the function of microsatellites in the genome has not been clearly identified, it is asserted that they are associated with some functional genes. Recently, several researchers have explored MHCassociated microsatellites, and they have been successfully used in several herbivore diversity studies (Fatima et al., 2008; Ligda et al., 2009; Salles et al., 2011). In the present study, BM1258 microsatellite variability was screened in the indigenous goat population (Salles et al., 2011).

The objective of this research was to determine the variability with altitude of BM1258, a highly polymorphic MHC-associated marker, in indigenous Southwest Chinese goats. We also investigated the effect of altitude on the dynamic development of the immunogenic region.

\section{MATERIAL AND METHODS}

\section{Animals and DNA isolation}

We analyzed 132 blood samples from four Chinese indigenous goat breeds. The first breed, Dazu Black (DZ) (50 individuals), is indigenous to Southwest China (geographic location: $37^{\circ} 180^{\prime}-29^{\circ} 52^{\prime} \mathrm{N}, 105^{\circ} 27^{\prime}-106^{\circ} 02^{\prime} \mathrm{E}$ ). This breed lives in an alpine habitat (between 267.1 and $934 \mathrm{~m}$ ) on the southeast of the Sichuan basin and in the western suburbs of Dazu, Chongqing 
region. The Hechuan White breed $(\mathrm{HC})$ (24 individuals) is indigenous to Hechuan in Chongqing (geographic location: $29^{\circ} 51^{\prime}-30^{\circ} 22^{\prime} \mathrm{N}, 105^{\circ} 58^{\prime}-106^{\circ} 40^{\prime} \mathrm{E}$ ). This breed lives in an alpine habitat (between 185 and $1284.2 \mathrm{~m}$ ). The Meigu breed (MG) (34 individuals) is indigenous to Sichuan in China (geographic location: $28^{\circ} 02^{\prime}-28^{\circ} 54^{\prime} \mathrm{N}, 102^{\circ} 53^{\prime}-103^{\circ} 21^{\prime} \mathrm{E}$ ). This breed also lives in an alpine habitat (between 800 and $2700 \mathrm{~m}$ ). The Tibetan breed (TG) (24 individuals) is indigenous to Linzhi on the Tibetan Plateau in Southwest China (geographic location: $26^{\circ} 73^{\prime}-36^{\circ} 53^{\prime} \mathrm{N}, 78^{\circ} 41^{\prime}-99^{\circ} 10^{\prime} \mathrm{E}$ ). Once again, this breed lives in an alpine habitat (higher than $4000 \mathrm{~m}$ ). For the detailed information about the samples in this study, we referred to the China National Commission of Animal Genetic Resources (2011) and the Animal Genetic Resources in Chongqing (2013). Blood samples were taken from the ulnar vein and stored in vacuum tubes containing ethylenediaminetetraacetic acid at $-20^{\circ} \mathrm{C}$ until required for examination. Genomic DNA was extracted from whole blood according to the Sambrook and Russell method (2001).

\section{Polymerase chain reaction (PCR) and genotyping}

The animals were genotyped via PCR amplification of the BM1258 marker using the forward primer BM1258-F: GTATGTATTTTTCCCACCCTGC, and the reverse primer BM1258-R: GAGTCAGACATGACTGAGCCTG. The BM1258 alleles were determined using PCR, as described by Salles et al. (2011). The amplification conditions comprised: an initial denaturation for $1 \mathrm{~min}$ at $94^{\circ} \mathrm{C}$, followed by 35 cycles at $92^{\circ}$ for $45 \mathrm{~s}, 56^{\circ} \mathrm{C}$ for $45 \mathrm{~s}$, and $72^{\circ} \mathrm{C}$ for $45 \mathrm{~s}$, with a 1.5 -h extension at $72^{\circ} \mathrm{C}$. Each PCR product (approximately $1-2 \mu \mathrm{L}$ ) was diluted with $10 \mu \mathrm{L}$ autoclaved distilled water for use in DNA genotyping. A 2- $\mu \mathrm{L}$ aliquot of the diluted product was mixed with $7.75 \mu \mathrm{L} \mathrm{Hi}$ $\mathrm{Di}^{\mathrm{TM}}$ Formamide and $0.25 \mu \mathrm{L}$ Gene Scan-500 $\mathrm{LIZ}^{\mathrm{TM}}$. The mixtures were heated at $94^{\circ} \mathrm{C}$ for $5 \mathrm{~min}$ and then immediately chilled on ice for 2 min. Genotyping was carried out on a 3130xl Genetic Analyzer (AB Applied Biosystems, USA).

\section{Genetic analysis of the population}

Gene and genotypic frequencies were estimated by direct counting. Expected homozygosity $\left(H_{\mathrm{E}}\right)$ and observed heterozygosity $\left(H_{\mathrm{O}}\right)$ were computed using the Microsatellite Toolkit (Park, 2001). Nei's unbiased estimates of genetic identity (genetic distance) and inbreeding coefficient $\left(F_{\text {IS }}\right)$ were also calculated using Arlequin software 3.5.1.3 (Excoffier and Lischer, 2010) and FSTAT 2.9.3.2 (Goudet, 1995), respectively.

\section{RESULTS}

In the Dazu Black goat ecotype, 11 alleles were identified for the BM1258 microsatellite. An allele of $99 \mathrm{bp}$ had the highest frequency (35\%), and alleles of $133 \mathrm{bp}$ had the lowest (2\%). We found 18 genotypes, of which 99/99 was the most frequent in this population. In the Hechuan White goats, 9 alleles were identified in the 24 samples tested. An allele of $109 \mathrm{bp}$ had the highest frequency (31\%); the lowest frequency alleles were 113 and $133 \mathrm{bp} \mathrm{(4 \% ).} \mathrm{Of} \mathrm{the} 15$ genotypes observed, 99/109 and 99/99 were the most frequent in the population. In the Meigu goats, 14 alleles were identified in the 34 samples tested. An allele of $109 \mathrm{bp}$ had the highest frequency (23\%); the lowest frequency alleles were $98,133,127$, and $107 \mathrm{bp}(2 \%)$. Of the 22 genotypes identified in this population, 101/101 was the most frequent (15\%). In the Tibetan goats, 8 alleles 
were identified in 71 samples tested. An allele of $109 \mathrm{bp}$ had the highest frequency (20\%); the lowest frequency alleles were 105, 111, and $107 \mathrm{bp}(2 \%)$. Of the 15 genotypes identified in this population, 109/109 was the most frequent (25\%).

Six alleles of BM1258 were shared within all four breeds: 99, 101, 103, 107, 109, and 111 bp. The results indicated a higher level of heterozygosity in all populations, with the proportion of observed heterozygous individuals ranging from 0.34 in the Dazu Black goats to 0.58 in the Hechuan White goats, and the expected level of heterozygosity ranging from 0.778 in the Dazu Black goats to 0.087 in the Meigu goats. The mean number of alleles ranged from 8 in the Tibetan goats to 14 in the Meigu goats. Detailed information on BM1258 allelic distribution is given in Figure 1 , and the relationship between the mean number of alleles and the altitude of the goats' habitats is given in Figure 2.

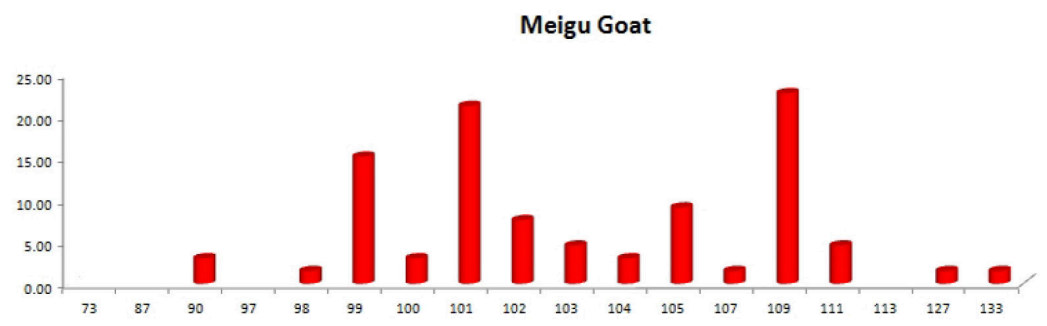

Dazu Black Goat
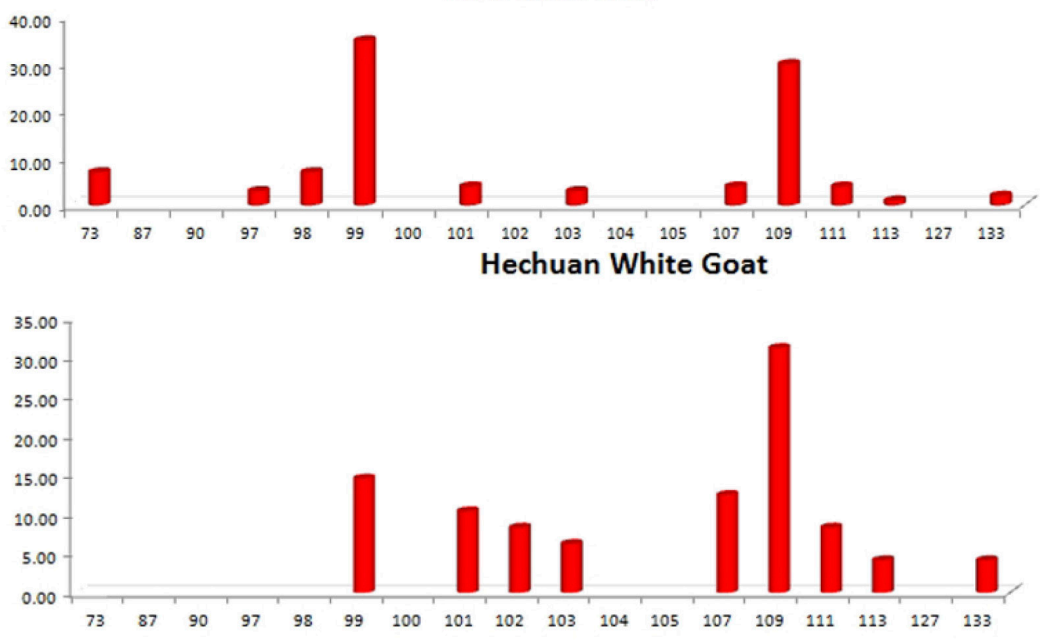

Tibetan Goat

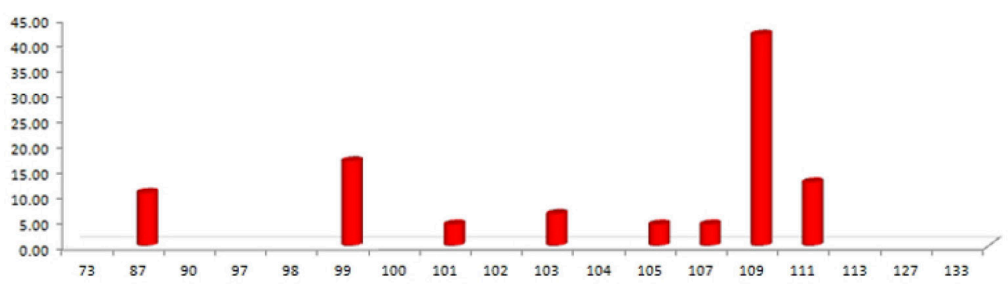

Figure 1. Allele distribution of BM1258 in each of the four different goat breeds. 


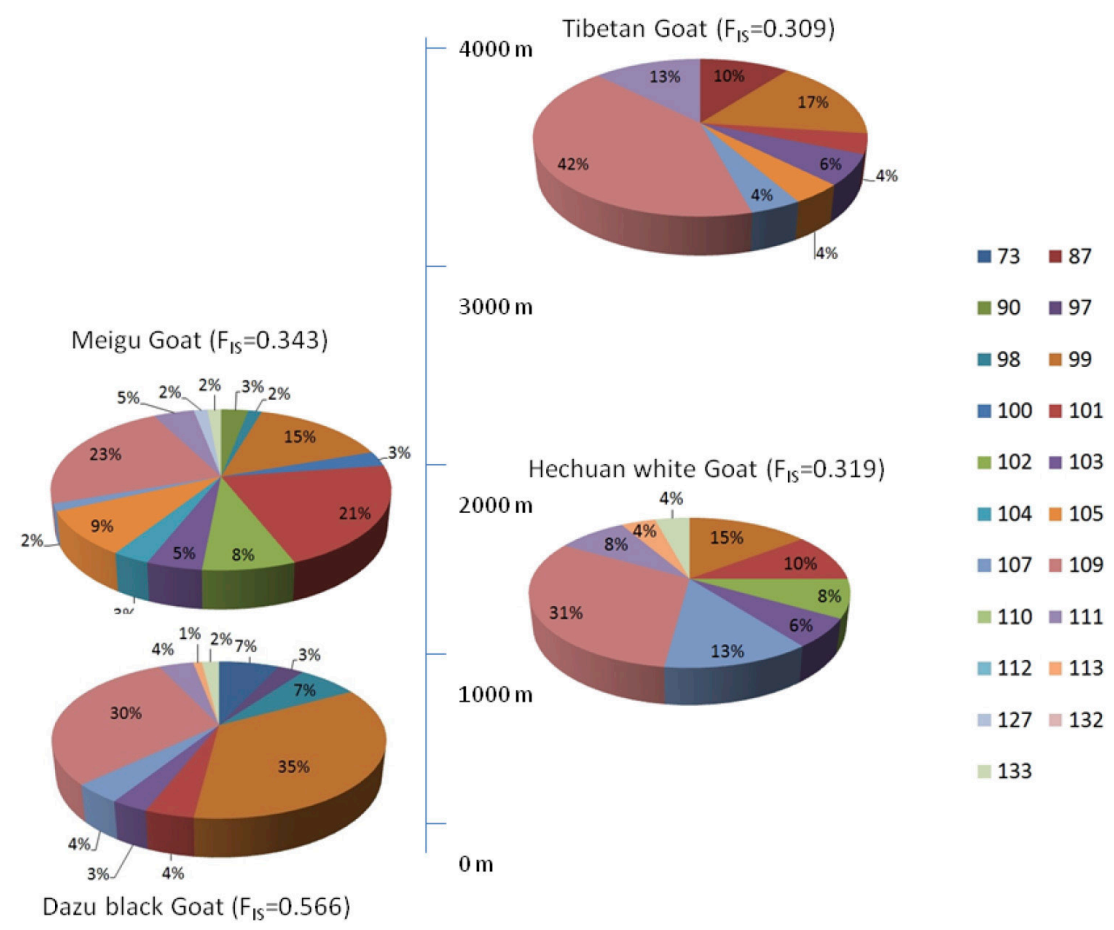

Figure 2. Pictorial diagram of diversity and population structure dynamic developing in each population at different altitudes.

Based on the genotyping data, the shortest Nei's genetic distance was 0.0079 , between the Meigu goats and the Hechuan White goats. The Dazu Black goats and the Meigu goats had the largest genetic distance (0.0401). In addition, the $F_{\text {Is }}$ within the whole population deviated significantly from zero, and the smallest $F_{\text {Is }}$ was in the Tibetan goats Table 1.

Table 1. Diversity and polymorphism measurements for BM1258 in different goat populations living at different altitudes.

\begin{tabular}{|c|c|c|c|c|c|c|c|c|c|c|c|c|}
\hline \multirow[t]{2}{*}{ Population } & \multirow[t]{2}{*}{ Sample size } & \multirow{2}{*}{$N_{\mathrm{A}}$} & \multicolumn{2}{|c|}{ Observed } & \multicolumn{2}{|c|}{ Expected } & \multirow[t]{2}{*}{$F_{\text {IS }}$} & \multirow[t]{2}{*}{$P$ value } & \multicolumn{4}{|c|}{ Population } \\
\hline & & & Homozygosity & Heterozygosity & Homozygosity & Heterozygosity & & & $\mathrm{DZ}$ & $\mathrm{HC}$ & MG & TG \\
\hline $\mathrm{DZ}$ & 50 & 11 & 0.660 & 0.340 & 0.212 & 0.778 & 0.566 & $0.010^{\star}$ & 0.0000 & & & \\
\hline $\mathrm{HC}$ & 24 & 9 & 0.417 & 0.583 & 0.149 & 0.851 & 0.319 & $0.010^{*}$ & 0.0249 & 0.0000 & & \\
\hline MG & 34 & 14 & 0.424 & 0.576 & 0.128 & 0.872 & 0.343 & $0.010^{*}$ & 0.0401 & 0.0079 & 0.0000 & \\
\hline TG & 24 & 8 & 0.458 & 0.542 & 0.221 & 0.779 & 0.309 & $0.010^{*}$ & 0.0276 & 0.0065 & 0.0328 & 0.0000 \\
\hline
\end{tabular}

$\mathrm{DZ}$ = Dazu Black; HC = Hechuan White; MG = Meigu; TG = Tibetan; $N_{\mathrm{A}}$ is the observed mean number of alleles; the indicative adjusted nominal level $(5 \%)$ for each $F_{\text {IS }}$ was 0.010 ; *means significant; after the column of $P$ values, along the right diagonal, are Nei's genetic distances between populations using BM1258 loci.

\section{DISCUSSION}

The MHC is commonly defined by serological reactions of erythrocytes with antibodies specific to the highly polymorphic MHC-specific antigens. Serological typing is fraught with the 
problem of cross-reactivity, which can complicate application of the technique to outbred populations (Fulton et al., 2006; Maina et al., 2015). The presence of novel haplotypes in different native breeds can contribute to this problem. With the advent of molecular biology tools, haplotypes of different class regions of the $\mathrm{MHC}$ region can now be determined using several methods, including two-dimensional gels, restriction fragment length polymorphism, DNA sequencing, single-strand conformation polymorphism, and sequence-specific PCR in various animals and tissues (Iglesias et al., 2003; Wilson et al., 2014; Ballingall et al., 2015; Lau et al., 2015). Unfortunately, these techniques are not always practical for all laboratories. To date, the microsatellite marker LEI0258 has provided a suitable method for the determination of MHC haplotypes in chickens (Chang et al., 2011; Chazara et al., 2013; E et al., 2014). However, as yet, there is no MHC-associated microsatellite equivalent to the LEI0258 of chickens for sheep or goats.

We studied BM1258 microsatellite variability in three distinct breeds of Chinese goat found at lower altitudes and one breed found at higher altitudes - the Tibetan goat. Our results indicate that the BM1258 microsatellite is highly polymorphic in the Chinese local breeds, but not in the Tibetan goat.

The number of alleles detected in each population was different. The indigenous breeds have been subjected to intensive selection according to specific economic breeding aims, which could explain the differences in allelic distribution. The differences in observed levels of heterozygosity were similar in the Dazu Black goats, the Hechuan White goats, and the Meigu goats, but smaller in the Tibetan goats. Theoretically, in the MHC region, observed heterozygosity should be far higher than the expected heterozygosity in local breeds. Researchers such as Worley (2010) found that on average, birds with heterozygote genotypes survived infection longer than homozygotes, and this effect was independent of genome-wide heterozygosity, estimated across microsatellite loci. MHC genes encode molecules that present peptide antigens to T-lymphocytes, thereby initiating the pathogen immune response. Whether domestic or wild, animals cannot avoid contact with pathogens during their lifetimes. Fluctuating selection may occur if pathogen load varies, and as such, the advantage of possessing a resistant allele also varies, maintaining variation (van Oosterhout, 2009). Finally, individuals that are heterozygous at MHC genes may have increased fitness compared with homozygous individuals, as they are potentially able to recognize and resist a larger variety of pathogen-derived peptides and have a higher survival rate. Several similar studies have been carried out in laboratory populations, where environmental variables were eliminated, showing significant effects of MHC heterozygosity on fitness (McClelland et al., 2003; Ilmonen et al., 2007; Worley et al., 2010).

However, the strange phenomenon observed in this study might be explained by the invasion of commercial breeds that decreases the population size of large indigenous goat breeds in China, resulting in some population events such as inbreeding or bottlenecks. This was also revealed by the $F_{\text {IS }}$ values in BM1258 within each population. The reason the value was smaller in the Tibetan goats was that their high-altitude living environment discourages the artificial introduction of commercial animals.

In addition, when we measured the mean number of BM1258 alleles in these populations, we found that the diversity decreased when the altitude of the animals' habitat increased. This phenomenon can be explained by the fact that at low altitudes or on plains, genetic material exchange and gene flow between different populations are easier.

We conclude that the microsatellite marker BM1258 can be used as a suitable method for MHC typing of local goat populations to estimate the diversity of MHC. The results will be helpful for genetic resource conservation as well as marker-assisted selection in goat breeding programs. 
It is noteworthy that the population diversity of the $\mathrm{MHC}$ genes is not only maintained by increasing the chance of selection, and balancing selection, but also by the relative effects of the social and geographical environment.

\section{Conflicts of interest}

The authors declare no conflict of interest.

\section{ACKNOWLEDGMENTS}

The authors alone are responsible for the content and writing of this article. Research supported by the Fundamental Research Funds for the Central Universities (\#SWU114023), the National Natural Science Foundation of China (\#31172195), and the 2013 Innovation TeamBuilding Program in Chongqing Universities (\#KJTD201334).

\section{REFERENCES}

Animal Genetic Resources in Chongqing (2013). Animal Genetic Resources in Chongqing. Chinese Agricultural Press, Chongqing.

Ballingall KT, Steele P, Lantier I, Cotelli M, et al. (2015). An ancient interlocus recombination increases class II MHC DQA diversity in sheep and other Bovidae. Anim. Genet. 46: 333-336.

Chang CS, Chen CF, Berthouly-Salazar C, Chazara O, et al. (2011). A global analysis of molecular markers and phenotypic traits in local chicken breeds in Taiwan. Anim. Genet. 43: 172-182.

Chazara O, Chang CS, Bruneau N, Benabdeljelil K, et al. (2013). Diversity and evolution of the highly polymorphic tandem repeat LEI0258 in the chicken MHC-B region. Immunogenetics 65: 447-459.

China National Commission of Animal Genetic Resources (2011). Animal Genetic Resources in China: Sheep and Goats. Chinese Agricultural Press, Beijing.

de Bakker PI, McVean G, Sabeti PC, Miretti MM, et al. (2006). A high-resolution HLA and SNP haplotype map for disease association studies in the extended human MHC. Nat. Genet. 38: 1166-1172.

E G, Sha R, Zeng S, Wang C, et al. (2014). Genetic variability, evidence of potential recombinational event and selection of LEI0258 in chicken. Gene 537: 126-131.

Edidin M (1988). Function by association? MHC antigens and membrane receptor complexes. Immunol. Today 9: 218-219.

Excoffier $L$ and Lischer HE (2010). Arlequin suite ver 3.5: a new series of programs to perform population genetics analyses under Linux and Windows. Mol. Ecol. Res. 10: 564-567.

Fatima S, Bhong CD, Rank DN and Joshi CG (2008). Genetic variability and bottleneck studies in Zalawadi, Gohilwadi and Surti goat breeds of Gujarat (India) using microsatellites. Small Ruminant Res. 77: 58-64.

Fulton JE, Juul-Madsen HR, Ashwell CM, McCarron AM, et al. (2006). Molecular genotype identification of the Gallus gallus major histocompatibility complex. Immunogenetics 58: 407-421.

Gaudieri S, Dawkins RL, Habara K, Kulski JK, et al. (2000). SNP profile within the human major histocompatibility complex reveals an extreme and interrupted level of nucleotide diversity. Genome Res. 10: 1579-1586.

Goudet J (1995). STAT (Version 1.2): A computer program to Calculate F-Statistic. J. Hered. 86: 485-486.

Gregersen JW, Kranc KR, Ke X, Svendsen P, et al. (2006). Functional epistasis on a common MHC haplotype associated with multiple sclerosis. Nature 443: 574-577.

Grossen C, Keller L, Biebach I, International Goat Genome Consortium, et al. (2014). Introgression from domestic goat generated variation at the major histocompatibility complex of Alpine ibex. PLoS Genet. 10: e1004438.

Iglesias GM, Soria LA, Goto RM, Jar AM, et al. (2003). Genotypic variability at the major histocompatibility complex (B and Rfp-Y) in Camperos broiler chickens. Anim. Genet. 34: 88-95.

IImonen P, Penn DJ, Damjanovich K, Morrison L, et al. (2007). Major histocompatibility complex heterozygosity reduces fitness in experimentally infected mice. Genetics 176: 2501-2508.

Klein J and Figueroa F (1986). Evolution of the major histocompatibility complex. Crit. Rev. Immunol. 6: 295-386.

Kloch A, Babik W, Bajer A, Siński E, et al. (2010). Effects of an MHC-DRB genotype and allele number on the load of gut parasites in the bank vole Myodes glareolus. Mol. Ecol. 19: 255-265. 
Lau Q, Chow N, Gray R, Gongora J, et al. (2015). Diversity of MHC DQB and DRB Genes in the Endangered Australian Sea Lion (Neophoca cinerea). J. Hered. 106: 395-402.

Li MH, Zhao SH, Bian C, Wang HS, et al. (2002). Genetic relationships among twelve Chinese indigenous goat populations based on microsatellite analysis. Genet. Sel. Evol. 34: 729-744.

Ligda CH, Altarayrah J, Georgoudis A and The ECONOGENE Consortium (2009). Genetic analysis of Greek sheep breeds using microsatellite markers for setting conservation priorities. Small Ruminant Res.83: 42-48.

Maina SM, Gitao CG and Gathumbi PK (2015). Hematological, serological and virological findings in sheep and goats experimentally infected with lineage III peste des petits ruminants virus isolates in Kenya. J. Exp. Biol. Agric. Sci. 3: 81-88.

McClelland EE, Granger DL and Potts WK (2003). Major histocompatibility complex-dependent susceptibility to Cryptococcus neoformans in mice. Infect. Immun. 71: 4815-4817.

Oliver MK, Telfer S and Piertney SB (2009). Major histocompatibility complex (MHC) heterozygote superiority to natural multiparasite infections in the water vole (Arvicola terrestris). Proc. Biol. Sci. 276: 1119-1128.

Park SDE (2001). Trypanotolerance in West African cattle and the population genetic effects of selection. Ph.D. Thesis, Genetic Department, University of Dublin, Ireland.

Rout PK, Joshi MB, Mandal A, Laloe D, et al. (2008). Microsatellite-based phylogeny of Indian domestic goats. BMC Genet. 9: 11.

Rout PK, Thangraj K, Mandal A and Roy R (2012). Genetic variation and population structure in Jamunapari goats using microsatellites, mitochondrial DNA, and milk protein genes. Sci. World J. 2012: 618909.

Salles Pde A, Santos SC, Rondina D and Weller M (2011). Genetic variability of six indigenous goat breeds using major histocompatibility complex-associated microsatellite markers. J. Vet. Sci. 12: 127-132.

Sambrook J and Russell DW (2001). Molecular cloning: a laboratory manual. 3rd edn. Cold Spring Harbor Laboratory Press, New York.

Satta Y, Li YJ and Takahata N (1998). The neutral theory and natural selection in the HLA region. Front. Biosci. 3: d459-d467.

The MHC Sequencing Consortium (1999). Complete sequence and gene map of a human major histocompatibility complex. Nature 401: 921-923.

Vahidi SM, Tarang AR, Naqvi AU, Falahati Anbaran M, et al. (2014). Investigation of the genetic diversity of domestic Capra hircus breeds reared within an early goat domestication area in Iran. Genet. Sel. Evol. 46: 27.

van Oosterhout C (2009). A new theory of MHC evolution: beyond selection on the immune genes. Proc. Biol. Sci. 276: 657-665.

Wei C, Lu J, Xu L, Liu G, et al. (2014). Genetic structure of Chinese indigenous goats and the special geographical structure in the Southwest China as a geographic barrier driving the fragmentation of a large population. PLoS One 9: e94435.

Westerdahl H, Asghar M, Hasselquist D and Bensch S (2012). Quantitative disease resistance: To better understand parasitemediated selection on major histocompatibility complex. Proc. Biol. Sci. 279: 577-584.

Wilson AB, Whittington CM and Bahr A (2014). High intralocus variability and interlocus recombination promote immunological diversity in a minimal major histocompatibility system. BMC Evol. Biol. 14: 273.

Worley K, Collet J, Spurgin LG, Cornwallis C, et al. (2010). MHC heterozygosity and survival in red junglefowl. Mol. Ecol. 19: 3064-3075. 